\title{
Hyperhidrosis - A Review on Idiopathic Unilateral Circumscribed Hyperhidrosis
}

\section{Dr. Pranami Kashyap, MD, Dr. Hari Kishan Kumar. Y, MD. DVL, Dr. Vivekananda, MD}

Department of Dermatology, Raja Rajeswari Medical College \& Hospital, Kambipura, Mysore Road, Bangalore. drkishanyadalla@rediffmail.com

*Corresponding Author: Dr. Hari Kishan Kumar. Y, MD. DVL, Professor of Dermatology, SKIN CARE CLINIC, Padma Nivasa, Raja Rajeswari Nagar, Bangalore.

\section{INTRODUCTION}

Hyperhidrosis is defined as an excessive sweating beyond the amount to return elevated body temperature to normal. ${ }^{1}$ In daily practice, it is said to be present when sweating is clinically noticeable under conditions where it would not be normally expected to occur, or occurs in excess. It can be classified as generalized and localized. ${ }^{2}$

Generalized hyperhidrosis is an excessive sweating covering a relatively large area $\left(>100 \mathrm{~cm}^{2}\right)$. It is comprised of primary and secondary forms. While primary or essential hyperhidrosis arises mainly from emotional and environmental factors; and is located in most cases, in the axilla, palms and soles; in the secondary form, an underlying neurologic or endocrinological disease is the cause of the diffuse sweating. Several drugs such as antipyretics, emetics, insulin, meperidine, anti-depressants such as fluoxetine, systemic cholinergic agents; as well as poisoning with arsenic, mercury and insecticides can cause generalized secondary hyperhidrosis. Systemic diseases ranging from infections (including febrile illnesses); cardiovascular disorders like shock and congestiveheartfailure; metabolicdiseaseslikediabetes mellitus, obesity, gout etc; endocrinal disorders such as hyperthyroidism and hyperpituitarism; several neurological conditions including dysautonomic states, brain lesions and spinal cord injury as well as neoplasms like pheochromocytoma, carcinoid syndrome etc can present with hyperhidrosis. ${ }^{3}$

Localised unilateral or segmental hyperhidrosis (LUH), on the other hand, is usually sharply demarcated and no larger than $10 \times 10 \mathrm{~cm}^{3}$ It was first described by Tarlov and Herz in 1947 and has an unclear pathogenesis. ${ }^{4}$ It may be idiopathic as will be discussed further, or associated with a primary cutaneous disorder, gustatory hyperhidrosis, primary focal hyperhidrosis, secondary to underlying malignancies, post-depilatory laser etc.

Idiopathic unilateral circumscribed hyperhidrosis is a rare disorder. It has none of the typical triggering factors found with essential hyperhidrosis, and the attacks can occur with no apparent cause even in sleep. ${ }^{5}$

\section{PATHOgENESIS}

Idiopathic LUH has an unclear pathogenesis. There are several case reports in medical literature suggesting association of LUH with various underlying disorders, such as Frey's syndrome ${ }^{6}$, cerebral infarction ${ }^{7}$, Buerger disease ${ }^{8}$,Holmes-Adiesyndrome ${ }^{9}$, Riley-Day syndrome or occurring secondary to intra-thoracic malignancies such as bronchial or pleural carcinomas ${ }^{10}$. Baskan EB et al. reported a case of LUH in a young male patient associated with type1 Neurofibromatosis, while Parslew R. et al found LUH secondary to an eccrine naevus in a young male patient ${ }^{11}$. It may also occur secondary to trauma, as reported by some authors., ${ }^{3,12}$ Kreyden et al postulated that in cases of trauma, LUH may be the result of a misdirected reconnection of the sympathetic nerve fiber network after injury, similar to Frey's syndrome, and not detectable by conventional neurological examination. However there have been several case reports in the available medical literature in which LUH had no underlying pathology and was termed idiopathic. ${ }^{5,13.14,15,16}$

\section{DiAgNosis}

Idiopathic LUH is a diagnosis of exclusion. It may be seen at any age of onset and in both men and women. Hence it is of extreme importance to elicit a detailed clinical history in terms of severity, extent, periodicity and duration of sweating, and also its impact on the 
individual's daily activities. Any past history of trauma, stroke, surgery, infection such as Herpes zoster etc must be ruled out. A complete physical examination of the patient is mandatory. Idiopathic LUH is usually has no particular trigger as opposed to essential hyperhidrosis, and occurs in a well circumscribed area of the body, usually not larger than $100 \mathrm{~cm}^{2}$.

A complete neurological evaluation is essential to rule out any hypo-/ anaesthesia over the lesion, thus ruling out Hansen's disease. Also both sensory and motor evaluation must be done if an underlying cerebral infarction, spinal cord or space-occupying-lesion of brain is suspected.

A detailed ophthalmological examination is also required in cases with a family history or with caféau-lait spots seen during physical examination, to look for Lisch nodules on the iris, as reported by Baskan EB et al. where LUH was associated with Type 1 Neurofibromatosis.

Gravimetric sweat test and Minor's iodine test can be done to evaluate the area of hyperhidrosis. The starch-iodine test was described by Minor in 1928 as a qualitative test to examine sudomotor function. A solution of tincture iodine is applied to the hyperhidrotic area and allowed to air-dry. Once dry, the area is dusted with starch. Sweating is then encouraged with or without triggering factors such as heat application, exercise, sauna etc. When sweat reaches the skin surface, the iodine reacts with the starch, thus causing a dramatic change in colour from yellow to dark blue, thus allowing visualization of the involved area.

Van de Kerkhof PC reported the use of a gravimetric method called Evaporative water loss (EWL) measurement on symmetrical test and control sites on the face, lower arms and upper legs to quantify and compare sweating in these sites. A cup of $2.8 \mathrm{~cm}$ diameter was placed over the test site and a constant stream of dry nitrogen was passed through it. The nitrogen and water vapour evaporating from the skin under the cup, was conducted via a Teflon tube into an electrolytic water analyser. To measure acetylcholine stimulation of EWL, $0.1 \mathrm{mg}$ acetylcholine in $0.1 \mathrm{ml}$ physiological saline was injected intracutaneously into each test area on the affected side of the body; and water vapour produced was recorded for 90 minutes and compared between the test and control sites. The length of time for which the EWL exceeded the maximum reading on the meter was taken as a measure of acetylcholine-stimulated EWL ${ }^{5}$. However, the use of gravimetric sweat test is limited in clinical practice.

It is also important to do a complete haemogram, hepatic and renal function tests, viral markers such as VDRL, chest radiograph, MRI and/or CT scan of brain and/or spine to rule out any underlying pathology.

Histopathological examination of a biopsy specimen taken from the site usually shows no abnormality. However, Parslew R et al found an increased number of eccrine sweat glands with normal sebaceous glands, consistent with an eccrine naevus. ${ }^{11}$

Hyperhidrosis can be scored on the Hyperhidrosis Disease Severity Scale (HDSS) as introduced by the Canadia Hyperhidrosis Advisory Committee:

1- Sweating is never noticeable and never interferes with daily activities.

2- Sweating is tolerable but sometimes interferes with daily activities.

3-Sweating is barely tolerable and frquently interferes with daily activities.

4- Sweating is intolerable and always interferes with daily activities.

Score- 1: Mild, 2: Moderate, 3-4: Severe ${ }^{2}$

This scale is very useful for assessment of relative improvement with therapy. Whereas a one-point improvement corresponds with approximately 50\% reduction in perspiration, a two-point improvement roughly corresponds with roughly $80 \%$ reduction.

Other subjective tools include the Dermatology Life Quality Index (DLQI) and Visual Analog Scale (VAS) ${ }^{17}$.

\section{TREATMENT}

While hyperhidrosis can have a negative impact on an individual's social life, in mild cases all that is needed is simple explanation and reassurance. Although a number of modalities of treatment have been tried out with variable success rates, it is still a difficult task to alleviate the problem completely.

- Systemic Treatment- Anticholinergic drugs such as Atropine or its analogues can successfully be used to treat idiopathic LUH. In a randomized single-blinded trial conducted by Wolosker et al. evaluating patient satisfaction with oxybutynin 
Hyperhidrosis- A Review on Idiopathic Unilateral Circumscribed Hyperhidrosis

versus placebo for treatment of palmar, plantar and axillary hyperhidrosis, more than $70 \%$ patients with palmar or axillary hyperhidrosis, and more than $90 \%$ of patients with plantar hyperhidrosis in the oxybutynin group noted significant to great improvement as compared to $27.3 \%$ and $13.4 \%$ patients respectively in placebo groups. QOL also showed $73.8 \%$ improvement in patients treated with oxybutynin while that in placebo group was only $13.8 \% \cdot{ }^{18}$ However use of anti-cholinergics is restricted due to adverse effects such as dryness of mouth, visual disturbances, glaucoma, urinary retention, hyperthermia and convulsions. ${ }^{2}$

Other alternatives such as Propantheline bromide, Methanthelinium bromide and Calcium Channel Blockers such as Nifedipine have also been tried. ${ }^{2}$

Takase Y. et al used oral Clonazepam at a dose of $1 \mathrm{mg}$ daily successfully to control all symptoms, which however recurred on discontinuing the drug. Systemic Amitriptyline has also been used in certain cases with variable outcomes.

- $\quad$ Topical Treatment- While Yadalla HK et al found topical $20 \%$ aluminium chloride very efficacious in controlling hyperhidrosis in a patient with idiopathic LUH, several other studies concluded that the results were not favourable, thus leading to persistence of symptoms. A case report stated that topical application of $5 \%$ poldine methysulphate in $70 \%$ alcohol in a case of Idiopathic LUH involving the forehead and scalp provided patial relief. ${ }^{19}$ Other agents such as 2\% Formaldehyde, 10\% Glutaraldehyde, 5\%-10\% methenamine gel, or $10 \%$ tannic acid in $70 \%$ alcohol have also been used for cases such as plantar hyperhidrosis; as these agents cause denaturation of proteins, thus leading to temporary blockage of sweat gland ducts. Topical application of anticholinergic agents has however proved to be mostly unsatisfactory. ${ }^{2}$

- Iontophoresis or introduction of ions of certain soluble salts into the tissues can be helpful in some cases, using various agents such as tap water, atropine sulfate, glycopyrrolate or a combination of ionic solvents such as an anticholinergic agent with aluminium chloride. The ions driven into the skin via pilosebaceous units and eccrine gland ducts are said to target areas with high electrolyte concentration due to the enhanced current flow, thus causing electrochemical coagulation of proteins and leading to disruption of eccrine gland function. Another theory postulates that tap-water iontophoresis (TWI), a safe, effective and inexpensive therapeutic option for idiopathic LUH, may also induce a functional disturbance of secretory mechanism of sweat glands by interrupting the stimulus-secretion coupling. Treatment of each palm or sole with 15-20 mA for 30 minutes daily induces significant cessation of hyperhidrosis in a week. ${ }^{2} \mathrm{~A}$ retrospective case note review of pediatric patients with localized hyperhidrosis undergoing TWI by Dagash $\mathrm{H}$ et al. shoed that there was significant decrease in mean HDSS following 7 sessions per patient. The most common adverse events noted were paraesthesia, pruritis, pain, erythema, dryness, abrasions and vesicle formation. A positive outcome was found in $84 \%$ patients $^{20}$.

- Botulinum, a neurotoxin produced by Clostridium botulinum, is being increasingly used now to treat cases of idiopathic LUH. Kreyden OP et al and Baskan EB et al found that treatment of their respective cases with Botulinum type-A toxin yielded good clinical response with symptomatic relief lasting for several months. ${ }^{4}$, ${ }^{12}$ The toxin blocks the release of acetyl choline at the neuromuscular junction by binding to specific membrane receptors on the presynaptic cholinergic neuron, causing paralysis of autonomic cholinergic fibres, including sympathetic fibres to sweat glands; and interfering with their secretory function. Both Botulinum type A and B can be used for treatment of hyperhidrosis. Each intra-dermal injection produces an area of dryness of around $1.2 \mathrm{~cm}$ in diameter for a number of months. Newer modalities such as 'Dermajet' and Iontophoresis can also be used to deliver Botulinum toxin percutaneously.

Basciani et al studied the efficacy and safety of Btx B in treatment of palmar hyperhidrosis and reported that following injection of 5000IU of Btx B into each palm, there was significant reduction of sweating levels from baseline at $4^{\text {th }}, 12^{\text {th }}$ and $24^{\text {th }}$ weeks. ${ }^{21}$ Side effects may range from mild pain and weakness of muscles around the injection site to serious iatrogenic botulism, thus highlighting the necessity for clinicians to have sufficient information of the agent prior to administration. 
- Nd:YAG 1064-nm laser can be successfully used in idiopathic LUH to cause a controlled destruction of eccrine and apocrine units, as reported by Goldman and Wollina in 17 cases of axillary hyperhidrosis. $^{22}$ Histological examination of laser-treated skin showed microvesiculation and decapitation to total vaporization of eccrine glands. ${ }^{17}$ It has proven to be a relatively easy, effective, non-invasive alternative to current treatment for this condition.

- Microwave Thermolysis is one of the newer treatment modalities, in which a microwave-based device is used to induce thermolysis of sweat glands at the dermo-hypodermal interface, while protecting the upper skin and structures beneath the subcutaneous fat. The microwave's selective heating of the dermis and glandular tissue more than subcutaneous fat is based on the relatively greater water content. ${ }^{17} \mathrm{~A}$ study by Hong et al using a FDA-approved microwave -based device for treatment of axillary hyperhidrosis in 31 patients showed that $94 \%$ of patients experienced atleast a one-point drop in HDSS on follow up, while 55\% reported a two-point or greater drop in HDSS. The average reduction in DLQI over the follow up period of 1, 3, 6 and 12 months was significantly more than 5 points. Adverse events related to this modality included site-related redness, swelling and discomfort, with a longer lasting abnormal skin sensation, neuropathy and weakness on the treated side ${ }^{23}$.

- Surgical treatment is reserved as the last resort for resistant cases of idiopathic LUH, ranging from excision of the sweat glands in the hyperhidrotic area, minimally invasive liposuction-curettage of sweat glands ${ }^{17}$, surgical curettage of subcutaneous tissue in the axillary vault to procedures as radical as sympathectomy. Despite having significant risk of infections, seroma, hematoma, necrosis, atrophic or hypertrophic scarring; surgical excision has significantly less incidence of relapse in the long run as compared to liposuctioncurettage $^{24}$. Although sympathectomy is a very effective modality in resolving symptoms of hyperhidrosis, a significant concern is postoperative development of compensatory hyperhidrosis in another anatomical region. Thus an alternative to sympathectomy is sympathotomy, which doesnot include ganglionectomy and disrupts axons post-synaptically after the T2 ganglion to avoid overzealous neural injury that may lead to neuronal regeneration causing severe compensatory hyperhidrosis. ${ }^{17}$ In a study by Atkinson et al, following endoscopic thoracic limited sympathotomy, post-operatively all the patients exhibited warm, dry palms; and after 3 months $96.6 \%, 69.2 \%$ and $39.8 \%$ patients demonstrated successful decrease of palmar, axillary and plantar hyperhidrosis respectively. ${ }^{25}$ Other surgical procedures such as supra-orbital nerve section have been found to provide relief in cases of idiopathic LUH involving the frontal $\operatorname{area}^{26}$.

\section{ConClusion}

Idiopathic Localized Unilateral Hyperhidrosis is a rare disorder with an unknown pathogenesis and a difficult management. It is important to rule out all underlying factors leading to the condition with appropriate investigations. While systemic and topical modes of treatment have been used for decades, newer options such as Botulinum injections, Laser, Microwave thermolysis are being increasingly practised with favorable clinical outcomes.

\section{REFERENCES}

[1] Sato K, Kang WH, Saga K, Sato KT. Biology of sweat glands and their disorders. II. Disorders of sweat gland function. J Am Acad Dermatol 1989;20:713-726.

[2] Gavvala M, Kabir BW, Nina N. Disorders of Sweat Glands. In: Sacchidanand S, editor. IADVL Textbook of Dermatology. $4^{\text {th }}$ ed. Mumbai (India): Bhalani; 2015. p. 1413-1467.

[3] YadallaHK,AmbikaH,ChawlaS.Acase ofidiopathic unilateral circumscribed hyperhidrosis. Indian J Dermatol 2013;58:163

[4] Baskan EB, Karli N, Baykara M, Cikman S, Tunali S. Localized unilateral hyperhidrosis and neurofibromatosis type 1: case report of a new association. Dermatology. 2005, 211: 286-289.

[5] van de Kerkhof PC, den Arend JA, Bousema MT, Stolz E. Localized unilateral hyperhidrosis. Br J Dermatol 1987;117:779-782.

[6] Kempermann G, Hemmer B, Lucking CH.A case of gustatory sweating and facial pain. Nervenarzt 1995;66:923-936. 
[7] Labar DR, Mohr JP, Nichlos FTD, Tatemichi TK. Unilateral hyperhidrosis after cerebral infarction. Neurology 1988;38:1679-1682.

[8] Boyvat A, Piksin G, Erdi H. Idiopathic unilateral localized hyperhidrosis. Acta Derm Venereol 1999;79:404-405.

[9] Coskun BK, Saral Y, Belirgen S, Oztürk P. Idiopathic unilateral hyperhidrosis with HolmesAdie syndrome: case report. J Dermatol. 2004; 31(6):473-476.

[10] Middleton WG. Bronchial carcinoma with pleural spread causing unilateral thoracic hyperhidrosis. BMJ 1976;2:563.

[11] Parslew R, Lewis-Jones M. Localized unilateral hyperhidrosis secondary to an eccrine nevus. Clin Exp Dermatol 1997;22:246-247.

[12] Kreyden OP, Schmid-Grendelmeier P, Burg G. Idiopathiclocalizedunilateralhyperhidrosis: Case report of successful treatment with botulinum toxin type A and review of the literature. Arch Dermatol 2001;137:1622-1625.

[13] Köse O, Baloglu H. Idiopathic unilateral circumscribed hyperhidrosis. Int J Dermatol 1997;36:209-210.

[14] Verbov J. Unilateral localized hyperhidrosis over face and scalp. Br J Dermatol 1974;90:470.

[15] Takase Y, Tsushimi K, Yamamoto K, Fukusako T, Morimatsu M. Unilateral localized hyperhidrosis responding to treatment with clonazepam. $\mathrm{Br} \mathrm{J}$ Dermatol. 1992;126:416.

[16] Ghali FE, Fine JD: Idiopathic localized unilateral hyperhidrosis in a child. Pediatr Dermatol 2000;1:25-28.

[17] Stashak AB, Brewer JD. Management of Hyperhidrosis. Clin Cosmet Investig Dermatol. 2014; 7:285-299.

[18] Wolosker N, de Campos JR, Kauffman P, PuechLeão P. A randomized placebo-controlled trial of oxybutynin for the initial treatment of palmar and axillary hyperhidrosis. J Vasc Surg. 2012;55(6):1696-1700.

[19] Cunliffe WJ, Johnson CE, Williamson DM. Localized unilateral hyperhidrosis: A clinical and laboratory study. Br J Dermatol. 1972;86:374378.

[20] Dagash H, McCaffrey S, Mellor K, Roycroft A, Helbling I. Tap water iontophoresis in the treatment of pediatric hyperhidrosis. J Pediatr Surg. 2017;52(2):309-312.

[21] Basciani M, Di Rienzo F, Bizzarrini M, Zanchi M, Copetti M, Intiso D. Efficacy of botulinum toxin type B for the treatment of primary palmar hyperhidrosis: a prospective, open, singleblind, multi-centre study. Arch Dermatol Res. 2014;306(5):497-503.

[22] Goldman A, Wollina U. Subdermal Nd-YAG laser for axillary hyperhidrosis. Dermatol Surg. 2008;34(6):756-762.

[23] Hong HC, Lupin M, O'Shaughnessy KF. Clinical evaluation of a microwave device for treating axillary hyperhidrosis. Dermatol Surg.2012;38(5):728-735.

[24] Wollina U, Köstler E, Schönlebe J, Haroske G. Tumescent suction curettage versus minimal skin resection with subcutaneous resection of sweat glands in axillary hyperhidrosis. Dermatol Surg. 2008;34(5):709-716.

[25] Atkinson JL, Fode-Thomas NC, Fealey RD, Eisenach JH, Goerss SJ. Endoscopic transthoracic limited sympathotomy for palmar-plantar hyperhidrosis: outcomes and complications during a 10-year period. Mayo Clin Proc. 2011;86(8):721-729.

[26] Tarlov MI, Herz E. Unilateral frontal hyperhidrosis relieved by supraorbital nerve section. JAMA. 1947;133:476-477.

Citation: Dr. Pranami Kashyap, Dr. Hari Kishan Kumar. Y, Dr. Vivekananda. Hyperhidrosis- A Review on Idiopathic Unilateral Circumscribed Hyperhidrosis. Archives of Dermatology and Skin Care. 2018; 1(1): 21-25.

Copyright: (c) 2018 Dr. Pranami Kashyap, Dr. Hari Kishan Kumar.Y, Dr. Vivekananda. This is an open access article distributed under the Creative Commons Attribution License, which permits unrestricted use, distribution, and reproduction in any medium, provided the original work is properly cited.

Archives of Dermatology and Skin Care V1 . I1 . 2018 